\title{
A New Approach for SAR Image Denoising
}

\author{
Murali Mohan Babu Y*, Subramanyam MV**, Giri Prasad MN*** \\ * JNTUA, Anantapur, AP, India. \\ ** ECE Dept. \& Principal, SREC, Nandyal, Kurnool, AP, India. \\ *** Dept. of ECE, JNTUA, Anantapur, AP, India.
}

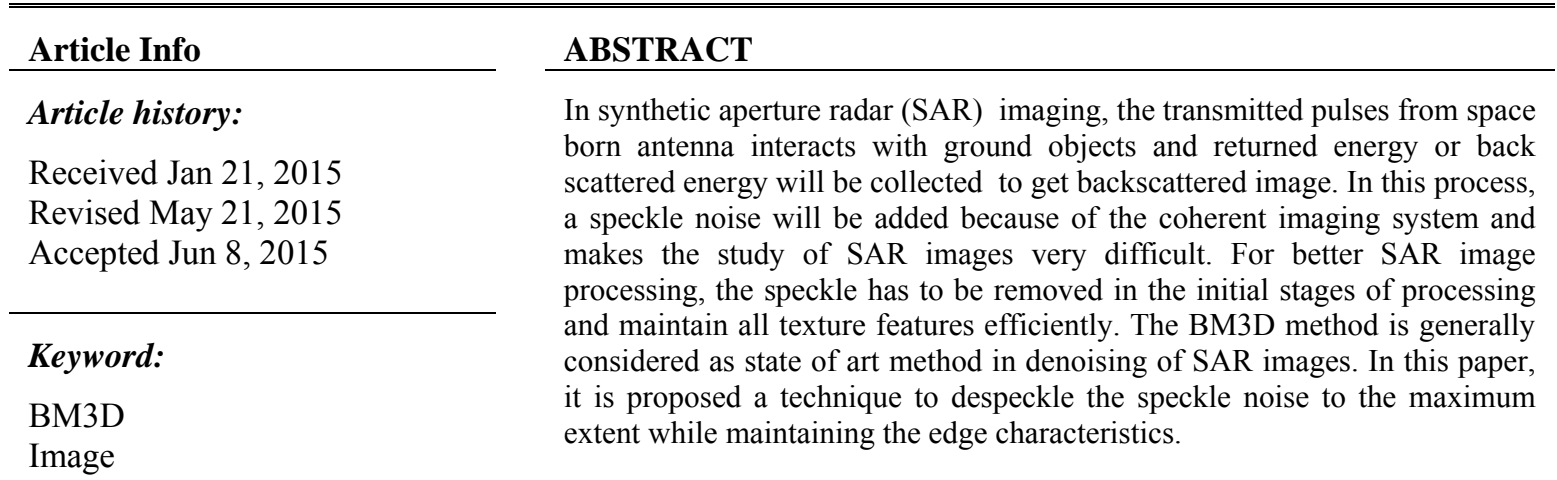

SAR

Speckle

Wavelet

Copyright (C) 2015 Institute of Advanced Engineering and Science. All rights reserved.

\section{Corresponding Author:}

Murali Mohan Babu Y,

Departement of Electronics and Communications Engineering,

Sri Venkateswara College of Engineering, Chittoor,

Andra Pradesh, India, 517127

Email: kisnamohanece@gmail.com

\section{INTRODUCTION}

Since its origin in the 1950 's, SAR has been developed into a mature technology and is now recognized as a highly successful imaging tool for environmental monitoring, forest cover mapping, glacier monitoring and military applications that require broad-area imaging at high resolutions. Synthetic aperture radar send microwave pulses towards the earth. These signals interact with ground objects and the backscattered radiation of the scene will be obtained at radar. Brighter areas are produced by stronger radar responses, due summation of microwave returns and darker areas are from weaker radar responses. The backscattered response generally depends on frequency or wavelength of the radar used, microwave pulse orientation or polarization, incidence angle of the microwave pulse and object on the earth.

Speckle is a common and special type of noise in all coherent imaging systems that is possible in SAR imaging system. The frequency domain filters mainly started with revolution from the invention of wavelets. The speckle has to be minimized to analyze the SAR image correctly. The removable of speckle plays critical and important role in preprocessing of any SAR system. A variety of spatial domain filters and transform domain procedures are available in SAR domain. But still there is possibility to despeckle further and further to achieve maximum reduction of speckle. Spatial domain filters like lee [1-4] and map filters [57] gave better despeckling results and are failed in preserving the edge details. Wavelet domain filters [8-11] have produced better response than spatial filters. That is the reason; the researchers have concentrated on transform domain filters [12-14].

A combination of transform domain and spatial domain image denoising algorithms is presented in BM3D algorithm. It consists of hard thresholding and wiener filter in wavelet domain. However, the smoothing of homogeneous areas and the preserving of edges are still not well balanced in these methods. 
But still the BM3D method is generally considered as state of art method in denoising of images and despeckling of SAR images [15]. The compressive sensing based 3D (CS3D) despeckling framework is comprised of three major steps; selection of subsets of pixels from SAR images, reconstruction of SAR image from each subset of pixels using CS theory, and statistical combining of multiple reconstructed images by employing selective 3D filtering. In this paper, we proposed a technique to despeckle the SAR images to the maximum extent while maintaining the edge characteristics. We compared our proposed method with state-of-art methods in terms of quality parameters like ENL, SSI, PSNR and ESI.

\section{DE-SPECKLING METHODS}

\subsection{BM3D Method}

The BM3-D algorithm contains two major steps. The first one contains transform domain hard thresholding to build a relatively clean image for estimating statistics, and the second one is having image denoising using wiener filtering in the same transform domain. In both the cases similarity between a group of blocks will be evaluated like in nonlocal approach. The size of the collected group will produce different responses. A combination of transform domain and spatial domain image denoising algorithms is presented in BM3D algorithm [16]. The selection of these thresholding levels vary the quality of output images and the selection of sub band in transform domain plays a major role in de noising the image.

The SAR-BM3D algorithm despeckles SAR images by combining the concepts of nonlocal filtering and wavelet hard shrinkage, which has a better capacity to preserve relevant details while smoothing homogeneous areas. However, the smoothing of homogeneous areas and the preserving of edges are still not well balanced in these methods. The BM3D method is generally considered as state of art method in denoising of images and despeckling of SAR images as Figure 1.

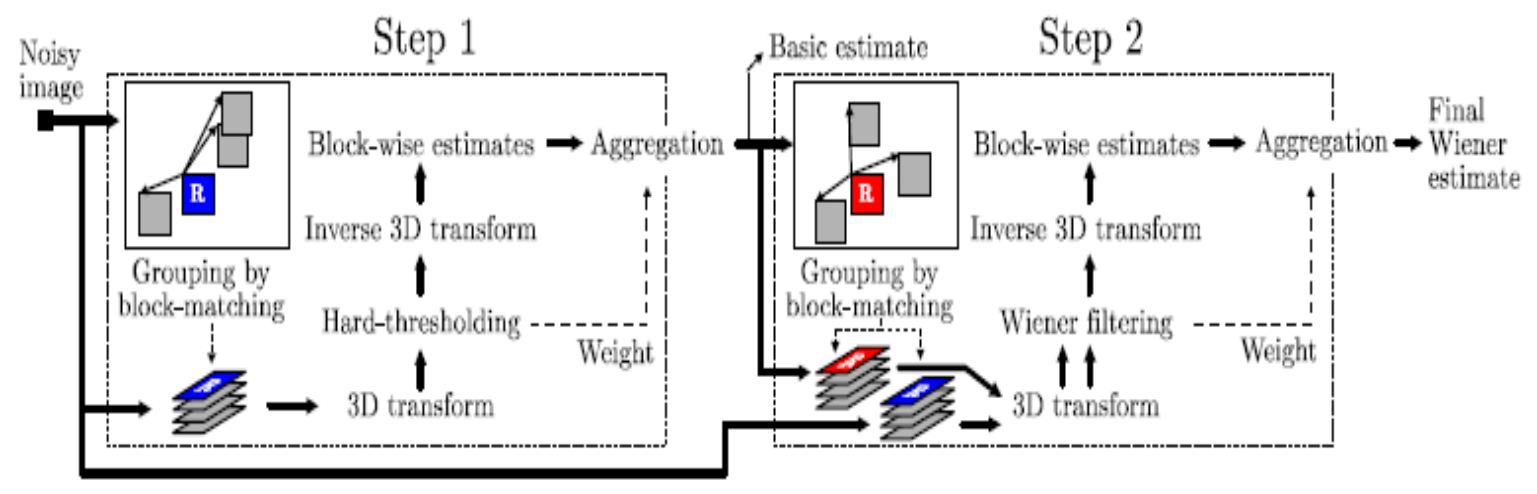

Figure 1. BM3D method

\subsection{CS3D Method}

The compressive sensing (CS) theory proved that any sparse signal or image can be reconstructed from samples fewer than number of elements in a signal or image [17]. These subsets are taken as measurement vectors in CS framework to obtain multiple SAR images by solving convex optimization problem. The pixel-wise averaging of multiple compressive reconstructed images would lead to better results compared to conventional despeckling techniques. In this work, employ selective 3 dimensional (3D) filtering of multiple reconstructed images to further improve despeckling results.

This despeckling framework is comprised of three major steps; selection of subsets of pixels from SAR images, reconstruction of image from each subset of pixels using CS theory, and statistical combining of multiple reconstructed images by employing selective 3D filtering, as shown in Figure 2. 


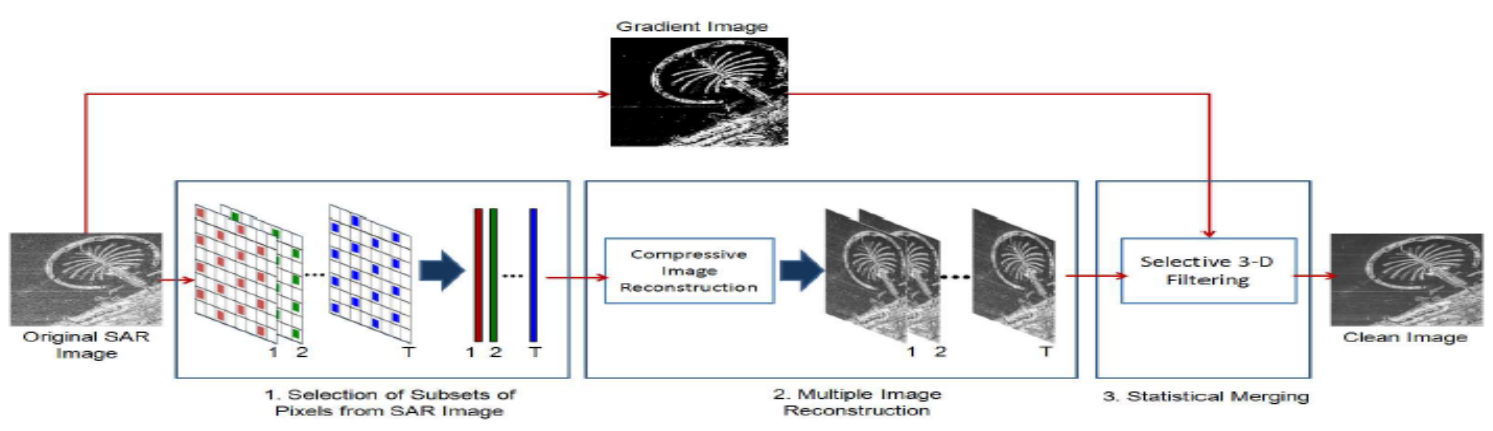

Figure 2. CS3D method

\subsection{Proposed Method}

The radar image will be cropped according to user's applications size. If the image is in geotiff format, generally it is noise free one; otherwise it is contaminated with noise. The necessary sized image will be given to non decimated wavelet transform and extract the all possible coefficients from it. After separating the coefficients, the required coefficients will be collected and they will be given to inverse non decimated wavelet transform.

The output image of previous step will be divided into different blocks with a fixed size. Each block will be compared with other blocks and euclidean distance will be calculated. If the distance is below a threshold value, then the block will be given to discrete wavelet transform and the coefficients will be extracted. Manipulation of coefficients will be done and an inverse discrete wavelet transform will be applied to reconstruct the image as shown in Figure 3.

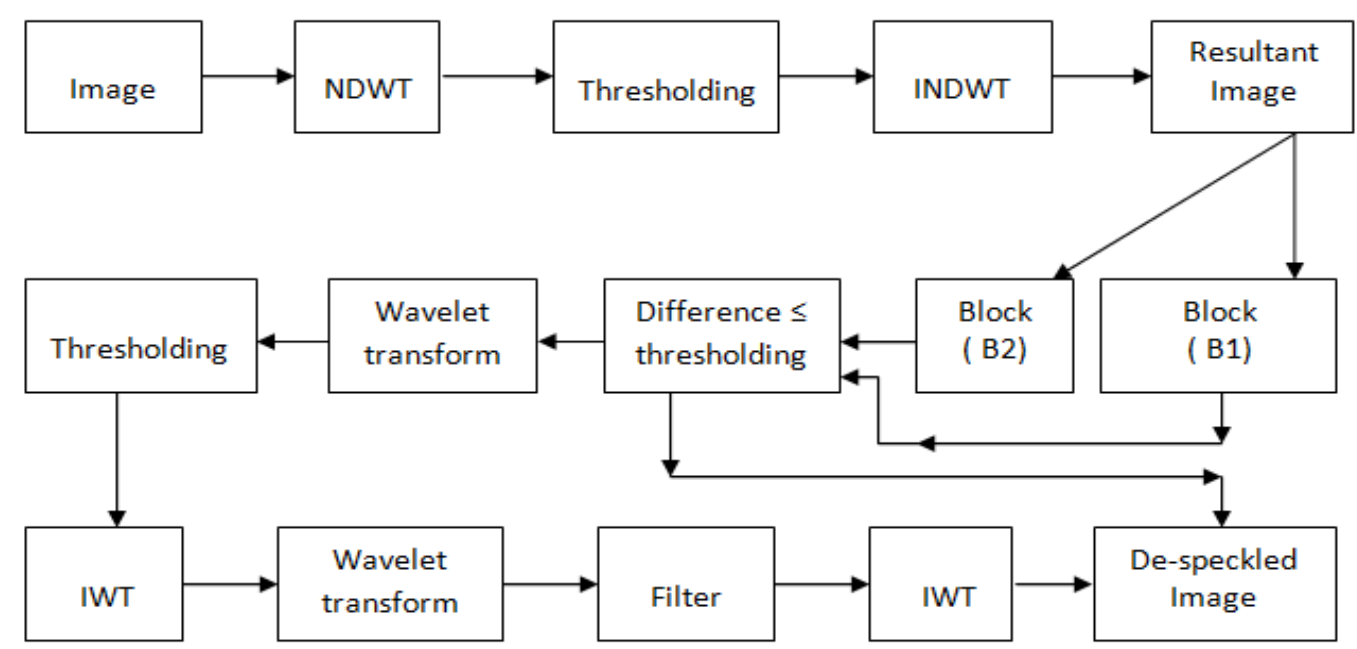

Figure 3. Proposed method

The last obtained image will be given to discrete wavelet transform once again and calculate all possible coefficients. A wiener filter will be applied on specific set of coefficient values and an inverse discrete wavelet transform will be applied to reconstruct the image.

The quality parameters like equivalent number looks (ENL), speckle suppression index (SSI), correlation coefficient (CC), edge saving or preserving index (ESI) and peak signal to noise ratio (PSNR) will be measured for all the output images of different latest despeckling methods along with proposed algorithm. 1. Peak Signal to Noise Ratio (PSNR): PSNR is the ratio between the maximum signal power and the corrupting noise power. It is the factor that judges whether a method is providing good denoising scheme or not. Higher the value means higher image quality.

$\operatorname{PSNR}=10 \log 10 *\left(2^{\mathrm{n}}-1\right) / \mathrm{MSE}$ 
2. Equivalent Number of Looks (ENL): Other than PSNR value, ENL value plays critical role in coherent systems like SAR processing. The ENL values speak about the efficiency in smoothing speckle noise of image over homogeneous areas.

$$
\mathrm{ENL}=(\text { mean } / \text { standard deviation })^{2}
$$

3. Coefficient of Correlation (CC): Correlation coefficient gives how far the two images correlated to each other, that means how far the despeckled image is near by the original image. It indicates the strength of linear relationship between the original $(x)$ and denoised mages $\left({ }^{\wedge} \mathrm{x}\right)$. If the value is near to 1 , then there exists stronger positive correlation between the $\mathrm{x}$ and ${ }^{\wedge} \mathrm{x}$ image.

$$
\mathrm{C}_{\mathrm{x},{ }^{\mathrm{x}}}=\mathrm{E}\left[\left(\mathrm{x}-\mu_{\mathrm{x}}\right)\left(\hat{\mathrm{x}}-\mu_{\wedge \mathrm{x}}\right)\right] / \sigma_{\mathrm{x}} \cdot \sigma_{\wedge \mathrm{x}}
$$

where $\mu_{\mathrm{x}}$ and $\mu_{\wedge_{\mathrm{x}}}$ are mean values of original and despeckled SAR images respectively and $\sigma_{\mathrm{x}}$ and $\sigma_{\wedge_{\mathrm{x}}}$ are standard deviations of original and speckle removed images respectively.

4. Speckle Suppression Index (SSI): The ratio of standard deviation to mean is used to measure the speckle strength in an image. Let $\mathrm{x}$ and ${ }^{\wedge} \mathrm{x}$ are original and despeckled SAR images. The SSI plays a critical role in radar image processing steps. It is defined as

$$
\mathrm{SSI}=\left[\left(\operatorname{var}\left({ }^{\wedge} \mathrm{x}\right)\right)^{1 / 2} / \operatorname{mean}\left({ }^{\wedge} \mathrm{x}\right)\right] *\left[\operatorname{mean}(\mathrm{x}) /(\operatorname{var}(\mathrm{x}))^{1 / 2}\right]
$$

It should be less than 1. Lower the value means higher the speckle reduction. Ideal value is zero.

5 Edge Save Index: Edge save index (ESI) explains the capability of the image how far the edge properties have been maintained.

$$
\begin{aligned}
& \operatorname{ESI}^{\mathrm{h}}=\sum_{i=1}^{m} \sum_{j=1}^{n-1}|\hat{x}(\mathrm{i}, \mathrm{j}+1)-\hat{x}(\mathrm{i}, \mathrm{j})| \quad / \quad \sum_{i=1}^{m} \sum_{j=1}^{n-1}|\mathrm{x}(\mathrm{i}, \mathrm{j}+1)-x(\mathrm{i}, \mathrm{j})| \\
& \mathrm{ESI}^{\mathrm{v}}=\sum_{j=1}^{n} \sum_{i=1}^{m-1}|\hat{x}(\mathrm{i}, \mathrm{j}+1)-\hat{x}(\mathrm{i}, \mathrm{j})| / \quad \sum_{j=1}^{n} \sum_{i=1}^{m-1}|\mathrm{x}(\mathrm{i}, \mathrm{j}+1)-x(\mathrm{i}, \mathrm{j})|
\end{aligned}
$$

where $\mathrm{x}$ is recovered image; $\mathrm{x}$ is original SAR image; $\mathrm{m}$ and $\mathrm{n}$ are the number of rows and columns of the SAR image.

\section{EXPERIMENTAL RESULTS}

All the filtering and transform domain techniques will be applied and tested mainly to the images of RISAT-1 sensor. Table 1 gives different quality factor values to test image. Generally ENL value plays a major role in deciding the despeckle algorithm to be used in microwave image processing. The higher the value of ENL, the better the performance of despeckling technique. Among all the techniques, the proposed method gives better ENL value.

Along with ENL value, SSI is an important parameter in the field of SAR imagery. Generally, speckle suppression index is less than 1. Lower the value means higher the speckle reduction. Among all the techniques, the proposed method gives least SSI value. That means maximum speckle has been removed.

Correlation coefficient gives how far the two images correlated to each other, that means how far the despeckled image is near by the speckled image. From table 2, we can say that our method gives better $\mathrm{CC}$ value.

Edge saving index is the critical parameter in any denoising method. Without better edge saving parameter, any method holds nothing. It can be observed that the proposed technique gives better ESI values among all methods. Similarly, the PSNR value that is obtained by the proposed method is high. It can be noted that, our despeckling technique performs better than other methods in quality factors terms of ENL, SSI, CC, ESI and PSNR. The despeckling results of the proposed technique and other methods to image are given in Figure 4 and Table 2. The PSNR values for different despeckling techniques on different RISAT-1 images have been given in Figure 5.

The area that we considered for despeckling is MAYKOP, RUSSIA. The central latitude and longitude values are 44.609 and 40.094 respectively. The characteristics of the scene have been given below in table 1. It is a RISAT-1 coarse resolution scanSAR mode CRS data 
Table 1. Characteristics of Data

\begin{tabular}{cc}
\hline Radar Carrier Frequency & $5.35 \mathrm{GHz}$ (C-band) \\
Incidence angle & $41.337 \mathrm{Deg}$ \\
Polarizations & $\mathrm{HH}$ \\
Datum & WGS 84 \\
Sensor height at the equator & $541 \mathrm{~km}$ \\
Revisit time (Orbit repeat cycle) & 12 days \\
Resolution & 36 meters \\
Mode & ASCENDING \\
Sensor Look & Right \\
Mean Local Time & $6 \mathrm{AM}$ \\
\hline
\end{tabular}

Table 2. Quantitative comparison of despeckling techniques for RISAT- 1 data

\begin{tabular}{cccccc}
\hline & ENL & SSI & CC & ESI & PSNR \\
\hline LEE & 17.74184 & 0.287047 & 0.511941 & 0.249804 & 10.76049 \\
WAVELET & 0.474352 & 0.584496 & 0.593618 & 0.170258 & 11.40477 \\
CURVELET & 0.565033 & 0.677036 & 0.73331 & 0.334237 & 17.94699 \\
PCA-LPG & 0.571852 & 0.433772 & 0.773526 & 0.274861 & 14.24952 \\
BM3D & 11.34283 & 0.52347 & 0.906364 & 0.624499 & 16.99552 \\
CS3D & 12.0902 & 0.07775 & 0.905526 & 0.620749 & 16.95302 \\
PROPOSED & $\mathbf{2 5 . 9 7 0 7 7}$ & $\mathbf{0 . 0 5 4 7 3 1}$ & $\mathbf{0 . 9 6 1 6 3 1}$ & $\mathbf{0 . 6 8 8 6 6 4}$ & $\mathbf{2 7 . 2 2 4 3 2}$ \\
\hline
\end{tabular}

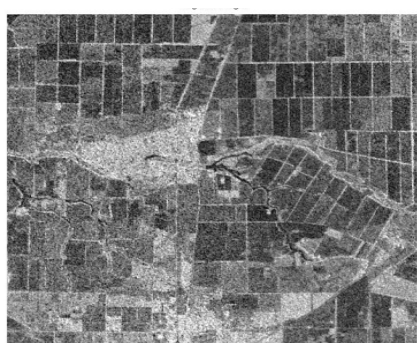

(a)

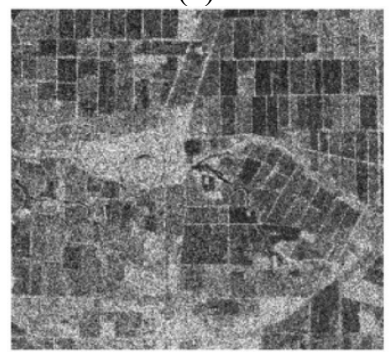

(d)

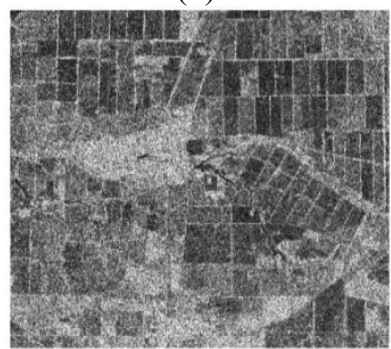

(g)

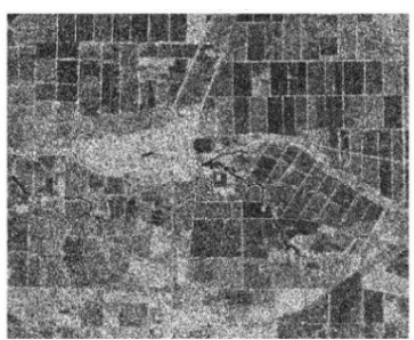

(b)

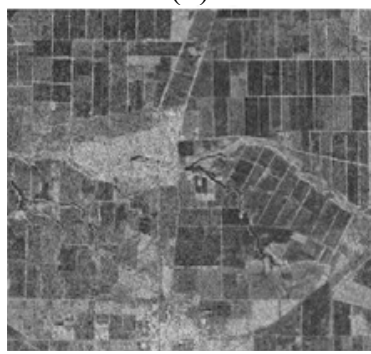

(e)

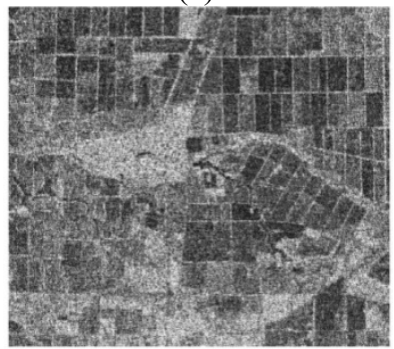

(h)

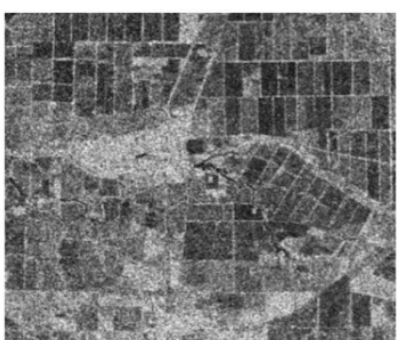

(c)

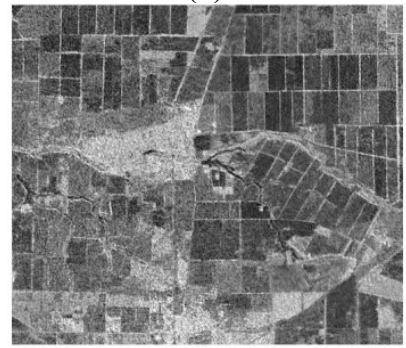

(f)

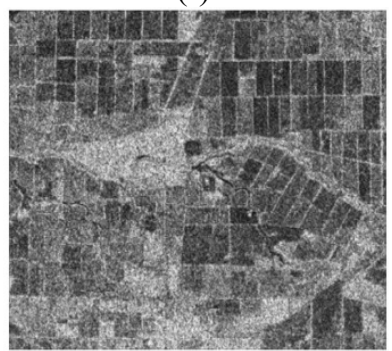

(i)

Figure 4. RISAT-1 dataset: Rural area near maykop city in Russia.(512*512) (a) Original, (b) Noisy, (c) Lee, (d) Wavelet, (e) Curvelet, (f)PCA-LPG, (g) BM3D, (h) CS3D and (i) Proposed.

The other study area is considered in the work is located on the east coast of India at a latitude of $17^{\circ} 42^{\prime}$ North and longitude of $83^{\circ} 23^{\prime}$ East and the time zone is GMT $+5: 30$. It is one of famous and major ports in India and the biggest port of Andhra Pradesh state. It is Visakhapatnam Port area. The table 3 shows the characteristics of the TerraSAR-X data. The despeckling results of the proposed technique and other methods to image are given in Figure 5 and Table 4. 
Table 3. Characteristics of TerraSAR-X Data

$\begin{array}{cc}\text { Radar Carrier Frequency } & 9.65 \mathrm{GHz} \text { (X-band) } \\ \text { Incidence angle range for: } & 20^{\circ}-45^{\circ} \text { full performance } \\ \text { Strip map/ScanSAR modes } & 20^{\circ}-55^{\circ} \text { full performance } \\ \text { SpotLight modes } & \left(15^{\circ}-60^{\circ} \text { accessible }\right) \\ \text { Polarizations } & \mathrm{HH}, \mathrm{VH}, \mathrm{HV}, \mathrm{VV} \\ \text { Pulse Repetition frequency } & 2.2 \mathrm{KHz}-6.5 \mathrm{KHz} \\ \text { Nominal orbit height at the equator } & 514 \mathrm{~km} \\ \text { Revisit time (Orbit repeat cycle) } & 11 \text { days } \\ \text { Inclination } & 97.44^{\circ}\end{array}$

$18: 00+/-0.25 \mathrm{~h}$ (local time)

Equatorial Crossing time

Table 4. Quantitative comparison of despeckling techniques for TerraSAR-X data

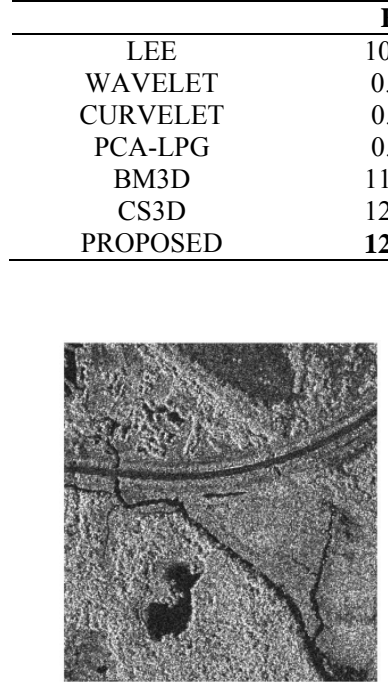

(a)

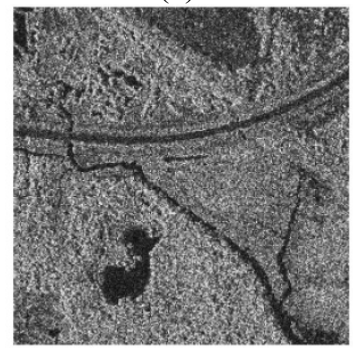

(d)

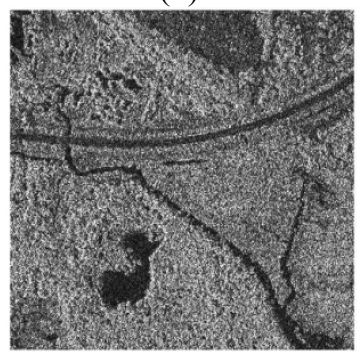

(g)

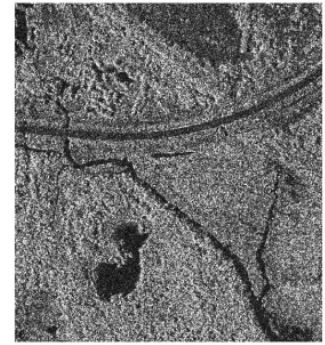

(b)

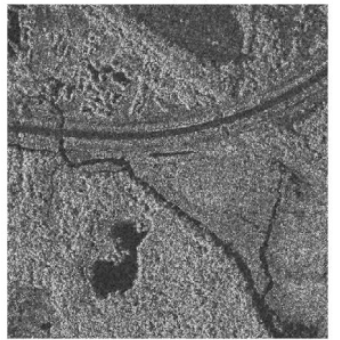

(e)

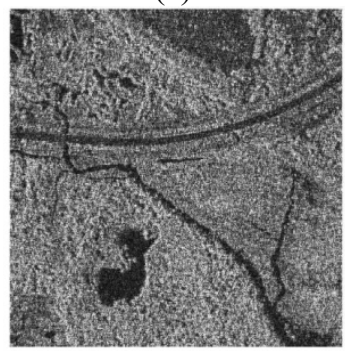

(h)

\begin{tabular}{ccc} 
CC & ESI & PSNR \\
0.5851 & 0.2619 & 12.6038 \\
0.6518 & 0.1656 & 13.2074 \\
0.8836 & 0.4415 & 18.3666 \\
0.9914 & 0.6940 & 25.7749 \\
0.9096 & 0.6113 & 18.4295 \\
0.9118 & 0.6172 & 18.5273 \\
$\mathbf{0 . 9 6 9 1}$ & $\mathbf{0 . 6 8 7 7}$ & $\mathbf{2 7 . 9 9 4 1}$ \\
\hline
\end{tabular}

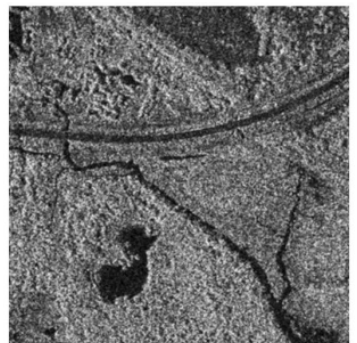

(c)

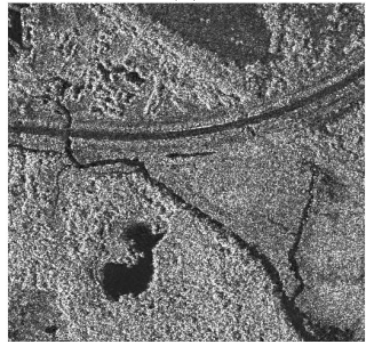

(f)

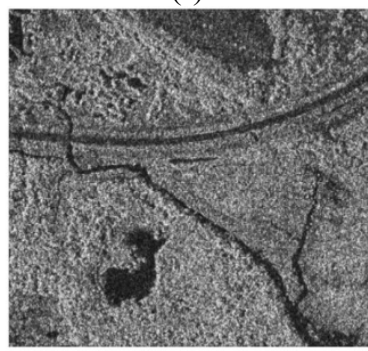

(i)

Figure 5. TerraSAR-X dataset: Visakhapatnam Port area in India. (512*512) (a) Original, (b) Noisy, (c) Lee, (d) Wavelet, (e) Curvelet, (f)PCA-LPG, (g) BM3D, (h) CS3D and (i) Proposed

The proposed method consists of BM3D method along with unsabsampled decimation. The decimation reduces the quality of the image that is to be denoised. It is because of down sampling at trasnitting end and upsampling at receiving end in wavelet transformation. The conversions of sampling are 
avoided in our proposed method. The PSNR values for different despeckling techniques on different RISAT1 images (Circular Fine resolution stripmap mode-Right Horizontal (Image-1), Circular Fine resolution stripmap mode-Right Vertical (Image-2), coarse resolution scanSAR-Horizontal Horizontal (Image-3), coarse resolution scanSAR- Horizontal Vertical (Image-4)) have been given in Figure 6.

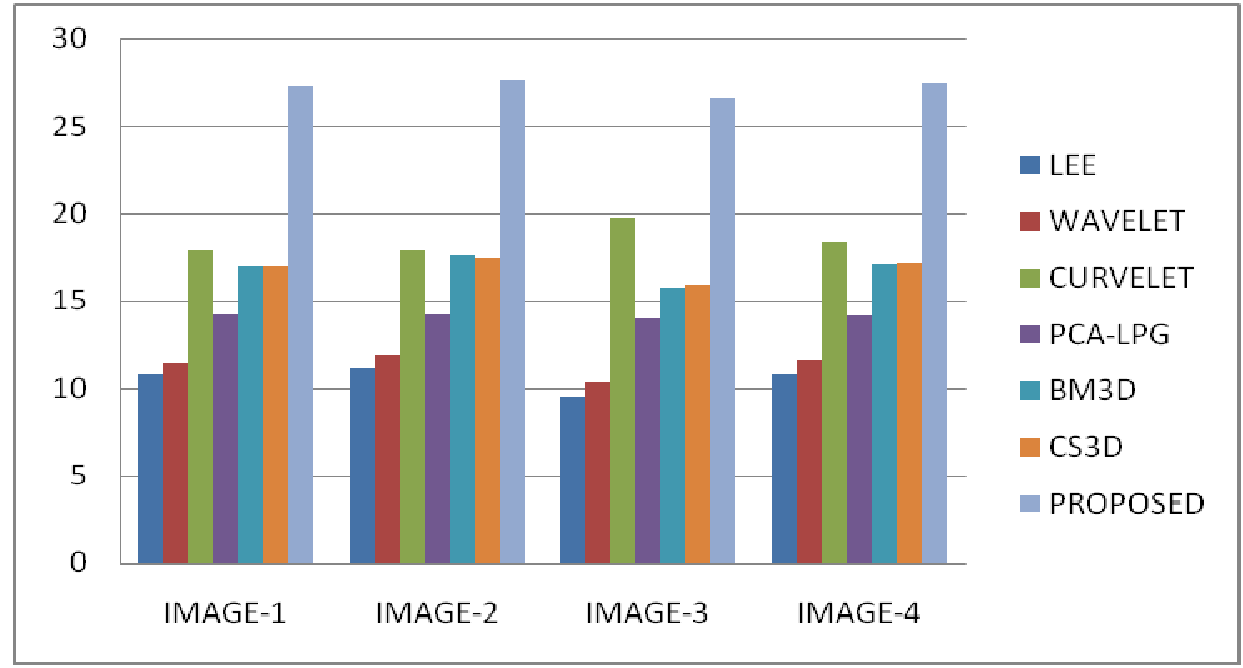

Figure 6. PSNR values for different images

\section{CONCLUSIONS}

We tested with different existing algorithms and proposed algorithm on different images of different modes of RISAT-1images and TerraSAR-X images. They have been tested with different noise levels (variances of $0.1,0.25$ and 0.5$)$ and different standard sizes $(256 * 256$ and $512 * 512)$. We considered different modes of RISAT-1 like coarse resolution scanSAR mode (CRS), medium resolution scanSAR mode (MRS), fine resolution stripmap mode (FRS) and different polarized images $(\mathrm{HH}, \mathrm{HV}, \mathrm{VH}, \mathrm{VV}, \mathrm{RH}$ and $\mathrm{RV})$ of RISAT-1 for testing. We also tested on different TerraSAR-X images and measured different quality parameters.

Even though the lee filter removes much speckle, it cannot preserve the edge details and it does not maintain the correlation with input image. It is a major drawback in statistical filters. The transform domain techniques convert the SAR image data into different frequency band in which the signal and noise are separated. The elimination of noise is simple at that moment. Because of this particular reason the transform based techniques will produce significant peak signal to noise ratios. Whereas the latest block based techniques like BM3D and CS3D are preserving the edge details and suppress the speckle better, but proposed method preserves edges and suppress the speckle much better than BM3D and CS3D techniques.

It is evidently observed that the proposed despeckling technique performs better than other latest methods in terms of quality factors like ENL, SSI, CC, ESI and PSNR.

\section{REFERENCES}

[1] Lee J.S. and E. Pottier, Polarimetric Radar Imaging from Basics to Applications, CRC Press, 2009.

[2] Lee J.S., L. Jurkevich, P. Dewaele, P. Wambacq, and A. Oosterlinck, Speckle filtering of synthetic aperture radar images: A review," Remote Sensing Reviews, Vol. 8, No. 4, 313-340, 1994.

[3] Frost V.S., J.A. Stiles, K.S. Shanmugan, and J.C. Holtzman, A model for radar images and its application to adaptive digital filtering of multiplicative noise", IEEE Transactions on Pattern Analysis and Machine Intelligence, Vol. 4, No. 2, 157- 166, Mar. 1982.

[4] Lopes A, Nezry E, Touzi R, and Laur H, "Maximum a posteriori speckle filtering and first order texture models in SAR images", in Proc. IEEE Int. Geosci. Remote Sens. Symp, vol. 3, pp. 2409-2412, 1990.

[5] Gagnon L and Jouan A, "Speckle filtering of SAR images-A comparative study between complex-wavelet-based and standard filters", in Proc. SPIE, pp. 80-91, 1997.

[6] Argenti.F and Alparone L, "Speckle removal from SAR images in the undecimated wavelet domain", IEEE Trans. Geosci. Remote Sens. vol. 40, no. 11, pp. 2363-2374, Nov. 2002. 
[7] Zhang L, Dong W, Zhang D, and Shi G, "Two-stage image denoising by principal component analysis with local pixel grouping", Pattern Recog., vol. 43, no. 4, pp. 1531-1549, Apr. 2010.

[8] Coupé P, Hellier P, Kervrann C, and Barillot C, "Bayesian non local means-based speckle filtering", in Proc. 5th IEEE Int. Symp. Biomed. Imaging, pp. 1291-1294, May 2008.

[9] Dabov K, Foi A, Katkovnik V, and Egiazarian K, "Image denoising by sparse 3D transform-domain collaborative filtering”, IEEE Trans. Image Process., vol. 16, no. 8, pp. 2080-2095, Aug. 2007.

[10] Parrilli S., M. Poderico, C.V. Angelino, and L. Verdoliva, A nonlocal SAR image denoising algorithm based on llmmse wavelet shrinkage", IEEE Transactions on Geoscience and Remote Sensing, Vol. 50, No. 2, 606-616, Feb. 2012.

[11] Goodman J.W, "Some fundamental properties of speckle", Journal of the Optical Society of America, vol. 66, no. 11, pp. 1145-1150, 1976.

[12] Lee J.S, Grunes M.R, and Mango S.A, "Speckle reduction in multipolarization, multifrequency SAR imagery", IEEE Transactions on Geoscience and Remote Sensing, vol. 29, no. 4, pp. 535-544, 1991.

[13] J.S. Lee, M.R. Grunes, D.L. Schuler, E. Pottier, and L. Ferro-Famil, "Scattering-model-based speckle filtering of Polarimetric SAR data", IEEE Transactions on Geoscience and Remote Sensing, vol. 44, no. 1, pp. 176- 187, 2006.

[14] Chang S.G, Yu B, and Vetterli M, "Spatially adaptive wavelet thresholding with context modeling for image denoising", IEEE Trans. Image Processing, vol. 9, pp. 1522-1531, Sept. 2000.

[15] Sveinsson J.R and Benediktsson J.A, "Speckle reduction and enhancement of SAR images using multi wavelets and adaptive thresholding", in Proc. SPIE Conf. Image and Signal Processing for Remote Sensing V, S. B. Serpico, Ed: EUROPTO Series, vol. 3871, pp. 239-250., 1999.

[16] Y. Murali Mohan Babu, M.V. Subramanyam \& M.N. Giriprasad "PCA based image denoising", Signal \& Image Processing: An International Journal (SIPIJ) Vol. 3, No. 2, 236-244, April 2012.

[17] Iqbal M., J. Chen W. Yang, P. Wang, and B. Sun, "SAR image despeckling by selective 3D filtering of multiple compressive reconstructed images," Progress In Electromagnetics Research, Vol. 134, No. 12, 209-226, 2013. 